\title{
Qualität in der forensischen Begutachtung erreichen, prüfen und aufrechterhalten
}

\author{
Henning Saß
}

Online publiziert: 8. Januar 2014

(C) Springer-Verlag Berlin Heidelberg 2013

\section{Achieve, test and maintain quality in forensic assessment}

Qualifizierung und Zertifizierung in der strafrechtlichen Begutachtung sowie im Maßregelvollzug bildeten das Schwerpunktthema im Band 6, Heft 4 unserer Zeitschrift Forensische Psychiatrie, Psychologie, Kriminologie vom November 2012. Schon damals war hinzuweisen auf die verschärfte Beobachtung unseres Faches in der Öffentlichkeit, auf die Abhängigkeit von Zeitgeist und politischen Strömungen, auf die immense Bedeutung der - in Stoßrichtung und Tenor sehr wechselhaften - Berichterstattung in Presse und Fernsehen sowie schließlich auf die Bedeutung von spektakulären Einzelfällen als Ausgangspunkt für stürmische Medienkampagnen.

Aktueller Anlass für Aufregung und Kritik waren seinerzeit die Geschehnisse um die terroristischen Gewaltakte eines ideologisch verrannten und möglicherweise wahnkranken Mannes in Skandinavien, um den es inzwischen wieder bemerkenswert still geworden ist. Mittlerweile ist in unserem Land ein ähnlich Aufsehen erregender Fall hinzugekommen, bei dem weniger das Anlassdelikt als vielmehr schwierige Umstände rechtlicher und gutachterlicher Art zu grundlegenden Zweifeln an Theorie und Praxis des psychiatrischen Maßregelvollzuges geführt haben. So problematisch im Übrigen auch manche publizistischen Auswüchse bei derartigen Problemfällen sein mögen, so begrüßenswert erscheint andererseits der Effekt, dass es bei

H. Saß $(\bowtie)$

Klinik für Psychiatrie, Psychotherapie und Psychosomatik,

Universitätsklinikum, RWTH Aachen,

52074 Aachen, Deutschland

E-Mail: hsass@ukaachen.de solchen Gelegenheiten zu einer grundlegenden Debatte und kritischen Überprüfung der geltenden Regelungen kommt. Dies kann dann zur Basis für notwendige Reformen werden, wie es sich gegenwärtig im Maßregelvollzug abzeichnet. Besonders wichtig erscheint es dabei, den Grundsätzen der Verhältnismäßigkeit von Freiheitsentzug und drohender Gefährdung ein größeres Gewicht zu geben.

Vergleichsweise deutlich weniger im Fokus des öffentlichen Interesses als die strafrechtlichen Bereiche stehen die Abläufe und Fallgestaltungen im Zivil- und Sozialrecht, obwohl auch diese für die betroffenen Individuen und gesamtgesellschaftlich von enormer Bedeutung sind. Dies betrifft zum einen zentrale ideelle Grundwerte unseres Lebens wie Menschenwürde, persönliche Freiheit und Selbstbestimmung, zum anderen aber auch handfeste ökonomische Konsequenzen etwa hinsichtlich der Kosten für die Krankenversicherung oder für Invalidität und Berentung. Aus solchen Gründen ist das Schwerpunktthema dieses Heftes der Qualifizierung und der Qualitätssicherung bei der gutachtlichen Tätigkeit in diesem Bereich gewidmet.

Am Beginn steht der Beitrag von Cording aus Regensburg, der neben der fundierten Darstellung grundsätzlicher Aspekte auch interessante Vorschläge zur Weiterentwicklung unserer Standards und Fortbildungsmaßnahmen enthält. Sodann behandelt Nedopil vom Münchener Lehrstuhl das von den praktischen Auswirkungen her besonders wichtige Gebiet der betreuungsrechtlichen Begutachtungsfragen, die im Hinblick auf die demografische Entwicklung in unserem Land stetig an Bedeutung gewinnen werden.

Eine Darstellung der Grundlagen für die sozialrechtliche Begutachtung liefern die Kollegen Foerster aus Tübingen und Dressing aus Mannheim, wobei insbesondere das in der Praxis ebenso wichtige wie vom theoretischen Konzept her vage Konstrukt der zumutbaren Willenstätigkeit eine hilfreiche Klärung erfährt. Beide Autoren widmen sich darü- 
ber hinaus in einem weiteren Beitrag dem derzeit besonders aktuellen Problem der Begutachtung posttraumatischer Belastungsstörungen, ein Thema, das trotz manch zeitgeistbedingter Überdehnung wichtige Grundsatzfragen der Kausalitätsbeurteilung berührt. Neuerdings führt dies bis zum Problem von Haftungsansprüchen an den Verursacher der traumatischen Schädigung hinsichtlich der Behandlungsund Folgekosten, ein Thema, das angesichts des komplexen Bedingungsgefüges bei Entstehung, Aufrechterhaltung und Therapie dieser Störungen außerordentlich vielschichtig erscheint.

Der Beitrag der Kollegen Sachs, Habermeyer und Ebner über die Situation der Qualifizierung und Qualitätskontrolle in der Schweiz ist zum einen von Bedeutung, weil dort gegenwärtig das curriculare System für die Weiterbildung in Forensischer Psychiatrie besonders sorgfältig aufgebaut wird, zum anderen bietet er aber auch eine interessante Vergleichsmatrix für unsere eigenen Regelungen. Diese werden dann in der den Themenkomplex abschließenden Arbeit von Müller aus Göttingen und Saimeh aus Lippstadt zusammengefasst, die beide die Sektion Forensik der Deutschen Gesellschaft für Psychiatrie und Psychotherapie, Psychosomatik und Nervenheilkunde (DGPPN) repräsentieren.
Auch hier finden sich, wie im ersten Beitrag, grundsätzliche Überlegungen zur künftigen Gestaltung der Konzepte für die Weiter- und Fortbildung in der forensischen Psychiatrie, die es weiter zu diskutieren gilt.

Dabei ist v. a. auf einen in Zukunft wichtigen Aspekt hinzuweisen, der in ähnlicher Form schon bei der strafrechtlichen Begutachtung herausgestellt wurde: Die Qualifizierung darf sich nicht nur auf den Erwerb des fachlichen Wissens konzentrieren, sondern es müssen auch Fähigkeiten und Kompetenzen in der praktischen Anwendung systematisch vermittelt werden. Darüber hinaus kann es nicht nur bei der einmaligen Zertifizierung bleiben, vielmehr benötigen wir, wie in anderen medizinischen Disziplinen, verbindliche Verfahren der kontinuierlichen Fortbildung, einschließlich damit verbundener Prüfungsschritte und „peer reviews“ im Sinne einer Rezertifizierung nach bestimmten Zeitabständen.

Als freier Beitrag folgt schließlich die Arbeit von Wößner aus Freiburg über die Methodik der Therapieerfolgsmessung, der zwar nicht direkt zum Schwerpunktthema gehört, aber durchaus in engem Bezug zur beständigen Aufgabe der Qualitätssicherung in unserem Fach steht. 